\title{
A Gait Paradigm Reveals Different Patterns of Abnormal Cerebellar Motor Learning in Primary Focal Dystonias
}

\author{
B. S. Hoffland • L. C. Veugen • M. M. H. P. Janssen • \\ J. W. Pasman • V. Weerdesteyn • B. P. van de Warrenburg
}

Published online: 3 September 2014

(C) Springer Science+Business Media New York 2014

\begin{abstract}
Accumulating evidence points to a role of the cerebellum in the pathophysiology of primary dystonia. The aim of this study was to investigate whether the abnormalities of cerebellar motor learning in primary dystonia are solely detectable in more pure forms of cerebellum-dependent associative motor learning paradigms, or whether these are also present in other motor learning paradigms that rely heavily on the cerebellum but in addition require a more widespread sensorimotor network. Twenty-six patients with various forms of focal dystonia and 10 age-matched healthy controls participated in a motor learning paradigm on a split-belt treadmill. By using reflective markers, three-dimensional kinematics were recorded using a 6-camera motion analysis system. Adaptation walking parameters were analyzed offline, comparing the different dystonia groups and healthy controls. Patients with blepharospasm and writer's cramp were significantly impaired on various adaptation walking parameters. Whereas results of cervical dystonia patients did not differ from healthy controls in terms of adaptation walking
\end{abstract}

Electronic supplementary material The online version of this article (doi:10.1007/s12311-014-0594-z) contains supplementary material, which is available to authorized users.

B. S. Hoffland - L. C. Veugen · J. W. Pasman •

B. P. van de Warrenburg $(\triangle)$

Department of Neurology, Donders Institute for Brain, Cognition and

Behaviour, Radboud University Medical Centre, Nijmegen, The Netherlands

e-mail: Bart.vandeWarrenburg@radboudumc.nl

L. C. Veugen • M. M. H. P. Janssen

Department of Biophysics, Donders Institute for Brain, Cognition and Behaviour, Radboud University Medical Centre, Nijmegen, The Netherlands

V. Weerdesteyn

Department of Rehabilitation, Donders Institute for Brain, Cognition and Behaviour, Radboud University Medical Centre, Nijmegen, The Netherlands parameters, differences in parameters of normal gait were found. We have here demonstrated abnormal sensorimotor adaptation with the split-belt paradigm in patients with blepharospasm and writer's cramp. This reinforces the current concept of cerebellar dysfunction in primary dystonia, and that this extends beyond more pure forms of cerebellumdependent associative motor learning paradigms. However, the finding of normal adaptation in cervical dystonia patients indicates that the pattern of cerebellar dysfunction may be slightly different for the various forms of primary focal dystonia, suggesting that actual cerebellar pathology may not be a primary driving force in dystonia.

Keywords Dystonia $\cdot$ Cerebellum $\cdot$ Sensorimotor adaptation

\section{Introduction}

Dystonia is a movement disorder characterized by involuntary muscle contractions resulting in abnormal postures and twisting movements. The basal ganglia are traditionally considered to be key player in the pathophysiology of dystonia, but accumulating evidence currently points to an additional role of the cerebellum. For example, eye blink classical conditioning (EBCC), a cerebellum-dependent paradigm of associative motor learning, was found to be abnormal in primary focal dystonia [1]. Cerebellar dysfunction in dystonia can firstly be due to pathological involvement of the cerebellum, but secondly also to a more compensatory engagement. The theory here then would be that the compensatory recruitment of the cerebellum is detrimental for other cerebellar tasks, such as associative motor learning. Interestingly, we recently showed that the EBCC deficits in primary dystonia can be modified by practice and also by direct noninvasive modulation of cerebellar excitability (through inhibitory continuous theta burst stimulation) [2]. This suggests that these functional deficits 
are reversible, which argues against a structural cerebellar defect.

The aim of this study was to investigate whether the abnormalities of cerebellar motor learning in primary dystonia are solely detectable in more pure forms of cerebellumdependent associative motor learning paradigms, such as EBCC, or whether these are also present in other motor learning paradigms that rely heavily on the cerebellum but in addition require more a widespread cerebral sensorimotor network. For this, we chose a motor learning paradigm on a split-belt treadmill [3]. Two types of gait adjustments are seen during split-belt walking: (1) direct reactive adjustments of walking parameters (e.g., stride length and time instance) to accommodate the novel difference in belt speeds and (2) adaptive feed forward adjustments in step length, time in double support, oscillation, and phasing parameters. Both cats with a transection of the spinal cord [4] and human infants [5] can make reactive feedback adaptations during split-belt treadmill locomotion, and these adjustments therefore are thought to predominantly or exclusively rely on spinal circuitry. Feed forward sensorimotor adaptation is, however, a more gradual form of motor learning used to adjust movements over minutes to hours in response to a certain perturbation, which leads to storage of a new motor pattern, which also persist as aftereffects when the perturbation is removed. The cerebellum is thought to be essential for this feed forward component as this paradigm challenges the ability to modify motor programs for adapting posture and locomotion through trial-and-error practice, which relies on the cerebellum [3]. We therefore expected to mainly pick up abnormalities in these adaptive feed forward adjustments if there are indeed more extensive cerebellar learning difficulties in dystonia patients.

Furthermore, the treadmill set-up also allowed us to assess normal gait and tandem gait walking to investigate possible subtle (cerebellar) gait abnormalities in dystonia patients. Prior studies have shown that in other movement disorders in which the clinical impression is that gait is normal, such as essential tremor, subclinical gait abnormalities can still be detected $[6,7]$.

\section{Methods}

Subjects Twenty-six dystonia patients (mean age 56.5 \pm 8.2 ; 13 males) and 10 age-matched healthy controls (mean age $54.8 \pm 7.8$ years; 4 males) participated in the study after giving their written informed consent. The patient group consisted of three subgroups: 10 cervical dystonia (CD) patients (mean age $54.0 \pm 7.4$; 4 males), 9 blepharospasm (BSP) patients (mean age 60.8 $\pm 8.6 ; 4$ males), and 7 writer's cramp (WC) patients $(55.3 \pm 8.8 ; 5$ males). The study was approved by the local ethics committee.
Patients who received botulinum toxin treatment were investigated in between treatments, e.g., when the effect of their last botulinum toxin injection was maximal. All patients demonstrated very good resolution of symptoms at this time. This was done to minimalize possible negative effects of head movement or involuntary blinking on task execution in patients.

Recording Twenty-six reflective markers were placed on anatomical landmarks according to the Vicon Plug-in- Gait model. Three-dimensional kinematics were recorded by a 6camera motion analysis system (Vicon Motion Systems, Oxford, UK) at a sample rate of $100 \mathrm{~Hz}$.

Paradigm A split-belt treadmill was used, consisting of two separate belts with independently controllable speeds (ForceLink BV, The Netherlands) $[5,8]$. Subjects walked for $2 \mathrm{~min}$ at a speed of $3.6 \mathrm{~km} / \mathrm{h}$ to investigate normal walking. After a short break, subjects were instructed to walk tandem (place one foot in front of the other, touching toe to heel) at $1.0 \mathrm{~km} / \mathrm{h}$ on a $3-\mathrm{cm}$ wide line that was projected on the treadmill. Two 1-min trials were collected.

Preferred walking speed was determined for each subject by two separate measurements on the treadmill. In addition, subjects filled out the Dutch version of the activities-specific balance confidence scale (ABC-NL) [9]. These two measurements were collected to assess normal gait in dystonia patients.

The split-belt experimental procedure consisted of different testing periods with the two belts either moving at same (tied belt) or different speeds (split belt). In tied belt configuration, both belts were set to move at slow $(1.3 \mathrm{~km} / \mathrm{h})$ or fast speed $(3.9 \mathrm{~km} / \mathrm{h})$. During split-belt walking, the right belt was set at high speed $(3.9 \mathrm{~km} / \mathrm{h})$ and the left belt was set at slow speed $(1.3 \mathrm{~km} / \mathrm{h})$.

We wanted to use a 3:1 ratio for split-belt walking as the largest adaptation and aftereffect are seen when the speed ratio between the two legs is greatest [10]. Previously, studies using the split-belt paradigm chose 0.5 and $1.5 \mathrm{~m} / \mathrm{s}$ for this $3: 1$ ratio. Given that some of our patients would not be able to sustain walking at these different speeds, we used a $1.3-3.9 \mathrm{~km} / \mathrm{h}$ $(0.36-1.08 \mathrm{~m} / \mathrm{s})$ ratio.

The paradigm consisted of a baseline period of $2 \mathrm{~min}$ walking at slow speed (S1), then subjects walked 2 min at fast speed (F), and again 2 min at slow speed (S2), all in the tied belts configuration. In the subsequent adaptation period (A), belts were switched to split-belt condition for $10 \mathrm{~min}$. For the post-adaptation $(\mathrm{P})$ period, subjects walked for $10 \mathrm{~min}$ at the slow tied belt configuration (Fig. 1).

Subjects were instructed not to look down at the belts while walking; during treadmill walking, one of the investigators stood at the front of the treadmill for safety reasons and to ensure participants did not look down during the experiment. The participants knew when the investigator changed the speed 


\begin{tabular}{|c|c|c|c|c|}
\hline L slow & L fast & L slow & L slow & L slow \\
\hline R slow & R fast & R slow & R fast & R slow \\
\hline S1 & F & S2 & A & PA \\
\hline
\end{tabular}

Fig. 1 Split-belt experimental set up: $S 1$ (slow walking 1) tied belt period of 2 min walking at $1.3 \mathrm{~km} / \mathrm{h} . F$ (fast walking) tied belt period of 2 min walking at $3.9 \mathrm{~km} / \mathrm{h}$. $S 2$ (slow walking 2) tied belt period of $2 \mathrm{~min}$ walking at $1.3 \mathrm{~km} / \mathrm{h} . A$ (adaptation) split-belt period (right belt $3.9 \mathrm{~km} / \mathrm{h}$ and left belt $1.3 \mathrm{~km} / \mathrm{h}$ ) of $10 \mathrm{~min} . P A$ (post-adaptation) tied belt period of $10 \mathrm{~min}$ walking at $1.3 \mathrm{~km} / \mathrm{h}$

of the treadmill; however, they were not aware of the details of this change during the split-belt experiment. Variable leg dominance, differences in preferred walking speed, and differences in side of dystonia symptoms could possibly influence adaptation, but as this was a first explorative study, we chose to use an identical setup for all groups of participants.

Data Analysis Marker position data were analyzed offline using custom written software in MATLAB (The MathWorks, Natick, USA). The four main walking parameters used in the analysis of split-belt adaptation are the following: step length symmetry, double support (DS) time symmetry, oscillation, and phasing $[8,11]$. The leg that was adapted on the slow belt will be referred to as the 'slow leg' and the leg on the fast belt as the 'fast leg' (even during tied belt walking). We also calculated the stance to swing time ratio, step width, foot angle, and an ataxia ratio [6] to investigate possible subtle gait differences during normal walking and tandem gait.

For the tandem gait recordings, the numbers of missteps (steps not hitting the projected line) were counted as well. Given that this number turned out to be very low, it was discarded from further analysis. For more information regarding the definitions and calculations of the above parameters, we refer to the supplementary methods.

To investigate the overall course of the split-belt experiment, we investigated walking over five different time periods. We therefore calculated the mean of the first five steps for slow walking, adaptation, and post-adaptation (S2, Abeginning, and P-beginning) and the mean of the last five steps of adaptation and post adaptation (A-end and P-end). Baseline values (mean of first five of last $30 \mathrm{~s} \mathrm{S2}$ ) were subtracted to normalize this data. To investigate if significant adaptation of these variables had occurred during split-belt walking, we first investigated whether P-beginning was significantly different from null in the different groups. Feed forward adjustments require practice to obtain and result in storage of a new movement pattern, observed as negative aftereffects after returning to the original condition. So, if there is no significant difference between baseline and post adaptation in a selected variable, no significant aftereffect is observed in this group. This indicates aberrant adaptation.

To compare the time course and development of step length adaptation, a rate of adaptation was calculated for step length symmetry. Walking parameters were averaged in bins of 10 steps for the first 210 steps of adaptation for each subject; corresponding to duration of $279.2 \pm 45.6 \mathrm{~s}$ on average, i.e., about half the adaptation period. To fairly compare this rate of adaptation, data was rescaled by their respective starting point, thus expressed as a percentage of the mean values over the first five steps of adaptation.

Data of one BSP patient was discarded from analysis of the split-belt experiment because of technical malfunctioning. Data of two CD patients was not available for normal walking analysis and data of one subject with $\mathrm{CD}$ for tandem gait analysis because of technical malfunctioning.

\section{Statistics}

Patient characteristics were tested with an ANOVA over GROUPs. Walking parameters during tandem gait and normal walking were tested using a one-way ANOVA with GROUP as between-subject factor (controls, WC, BSP, and CD).

Adaptation aftereffects were first investigated with a one sample $T$-test in which P-beginning was tested against null to see if significant adaptation occurred. After this, a repeated measures ANOVA was performed on the five different walking periods with between-subject factor GROUP (controls, WC, BSP, CD) and within-subject factor PERIOD (S2, Abeginning, A-end, P-beginning, P-end).

Finally, for the adaptation rate, we tested the number of steps (in bins of 10 steps) participants took to adapt to $50 \%$ of their starting point using a one-way ANOVA with betweensubject factor GROUP. If $50 \%$ adaptation was not reached after 210 steps, this maximum value was used in the analysis.

In the case of a significant main effect, post hoc comparisons were made using LSD tests. Pearson's correlation coefficients were calculated for all walking variables versus the ABC-NL outcomes. Significance was set at $p$ less than 0.05, unless stated otherwise.

\section{Results}

Participant Characteristics There were no GROUP differences in age between controls and the patient groups $(F(3)=$ 
1.194, $p=0.327)$. The same applies to the proportion of male subjects $\left(\chi^{2}(3)=2.09, p=0.55\right)$ (Table 1$)$.

Preferred Walking Speed For the preferred walking speed, there was a GROUP effect $(F(3)=6.860, p=0.001)$. The preferred walking speed in CD and BSP patients was lower compared with controls ( $p=0.005$ and $p=0.001$, respectively) and compared with patients with WC $(p=0.013$ and $p=0.003$, respectively) (Table 1).

Balance Confidence Also the ABC-NL yielded significant GROUP effects $(F(3)=9.791, p<0.001)$. Post-hoc tests revealed reduced confidence in one's own balance in BSP versus all other groups $(p \leq 0.005)$ and in $\mathrm{CD}$ versus controls $(p=0.046)($ Table 1$)$.

Normal Walking No significant differences were found for step width, swing/stance ratio, or step length symmetry. However, for the normal walking measures, there was a significant difference between GROUPS for the ataxia ratio $(p=0.035$, $F(3)=3.251)$. According to post hoc tests, patients with BSP had a more variable gait than those with WC $(p=0.004)$.

Tandem Gait No GROUP effects were found for any of the tandem gait walking measures (step width, swing/stance ratio, foot angle, ataxia ratio, or step length symmetry).

Aftereffect To investigate if adaptation had occurred, the presence of an aftereffect was investigated by testing P-beginning against null using a one sample $T$-test for different groups.

In healthy controls, P-beginning significantly differed from zero for all variables (step length $t(9)=4.51, p=0.001$, double support time $t(9)=3.46, p=0.007$, oscillation $t(9)=-2.92$, $p=0.017$, and phasing $t(9)=-6.55, p<0.001)$. Also in $\mathrm{CD}$ patients, P-beginning significantly differed from zero for all variables (step length $t(9)=6.14, p<0.001$, double support time $t(9)=6.14, p<0.001$, oscillation $t(9)=-3.09, p=0.013$, phasing $t(9)=3.28, p=0.010)$. In BSP patients, P-beginning did not significantly differ from zero for step length $(t(7)=$ $2.35, p=0.051)$, for double support time $(t(7)=1.15, p=0.29)$, and for oscillation $(t(7)=-1.92, p=0.097)$. In BSP patients, Pbeginning significantly differed from zero for phasing $(t(7)=$ $-3.30, p=0.013)$. In WC patients, P-beginning did not significantly differ from zero for double support time $(t(6)=$ $-0.48, p=0.65)$ or for phasing $(t(6)=-1.24, p=0.27)$. In WC patients, P-beginning significantly differed from zero for step length $(t(6)=6.35, p=0.001)$ and oscillation $(t(6)=-2.68$, $p=0.037$ ) (Fig. 2).

Walking Parameters for the Five Periods Running a rANOVA over the five different periods did not result in significant group or PERIOD*GROUP interactions for steplength symmetry, oscillation, or phasing. For DS symmetry, both PERIOD*GROUP $(F(3)=3.547, p=0.026)$ and $\operatorname{GROUP}(F(3)=5.157, p=0.005)$ were significant. When testing the periods separately, there was only a significant GROUP effect for DS time symmetry on A-end, P-beginning, and P-end $(F(3)=3.994, p=0.016 ; F(3)=3.242, p=0.035$; $F(3)=4.902, p=0.007)$. For A-end, significant differences existed between WC and all three other groups $(p<0.02)$; for P-beginning, between WC and controls $(p=0.013)$ and $\mathrm{CD}$ $(p=0.016)$; and for P-end between controls and all other groups $(p<0.025)$ (Fig. 3).

\section{Adaptation Rate}

There was no difference in the mean first five steps of step length symmetry between all groups $(F(3)=0.167, p=0.918)$. There was a significant GROUP effect for the number of steps it took for participants to adapt to $50 \%$ of their starting point $(F(3)=4.311, p=0.012)$. LSD post hoc tests showed differences between controls and WC $(p=0.018)$ and BSP $(p=0.017)$, between BSP and CD $(p=0.014)$ and between WC and CD $(p=0.016)$. Adaptation was thus significantly slower in patients with BSP and WC compared to the healthy controls and to CD patients (Fig. 4).

\section{Discussion}

There are other studies that addressed cerebellar learning capacities in patients with dystonia, and the tasks used were eye-blink classical conditioning (EBCC; a form of cerebellum-dependent associative motor learning) and
Table 1 Participant characteristics

\begin{tabular}{llllll}
\hline & Controls & All patients & WC & BSP & CD \\
\hline Mean \pm SD & & & & & \\
$\quad$ Number of subjects & 10 & 26 & 7 & 9 & 10 \\
Male [\%] & 40 & 50 & 71 & 44 & 40 \\
Age [years] & $54.8 \pm 7.77$ & $56.5 \pm 8.2$ & $55.3 \pm 8.8$ & $60.8 \pm 8.6$ & $54.0 \pm 7.4$ \\
Pref. walking speed [km/h] & $4.6 \pm 0.5$ & $3.9 \pm 0.9$ & $4.6 \pm 0.8$ & $3.4 \pm 0.6$ & $3.7 \pm 0.9$ \\
Balance scale [\%] & $99.0 \pm 0.9$ & $90.9 \pm 9.5$ & $97.8 \pm 2.1$ & $84.0 \pm 11.0$ & $93.0 \pm 6.9$ \\
\hline
\end{tabular}



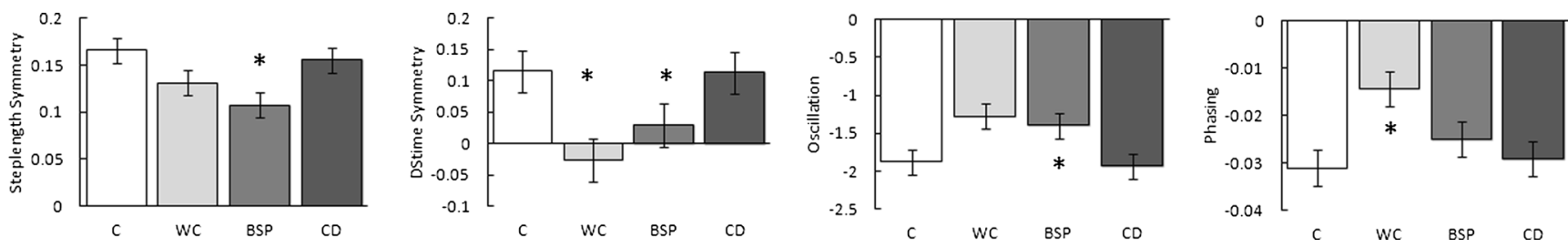

Fig. 2 Aftereffect. Asterisk indicates P-beginning did not significantly differ from null

sequence learning. The observed EBCC deficits were shown to be modifiable [2] and the cerebellum was shown to be hyperactive during sequence learning [12]. These studies let us believe that cerebellar abnormalities in dystonia are functional and possibly even compensatory in nature. In line with this is the lack of overt clinical sings of cerebellar dysfunction, including gait, in patients with primary dystonia. However, sensorimotor adaptation has to our knowledge never been studied in primary dystonia. The cerebellum is thought to be essential for sensorimotor adaptation, and the paradigm we have used here challenges the ability to modify motor programs for adapting posture and locomotion through trial-anderror practice, which relies on the cerebellum.

We investigated feed forward sensorimotor adaptation with the split-belt paradigm [3] to study the cerebellar control of locomotion in various forms of primary, focal dystonia. We deliberately chose to study various types of focal dystonia to comment on the issue of convergence or divergence of
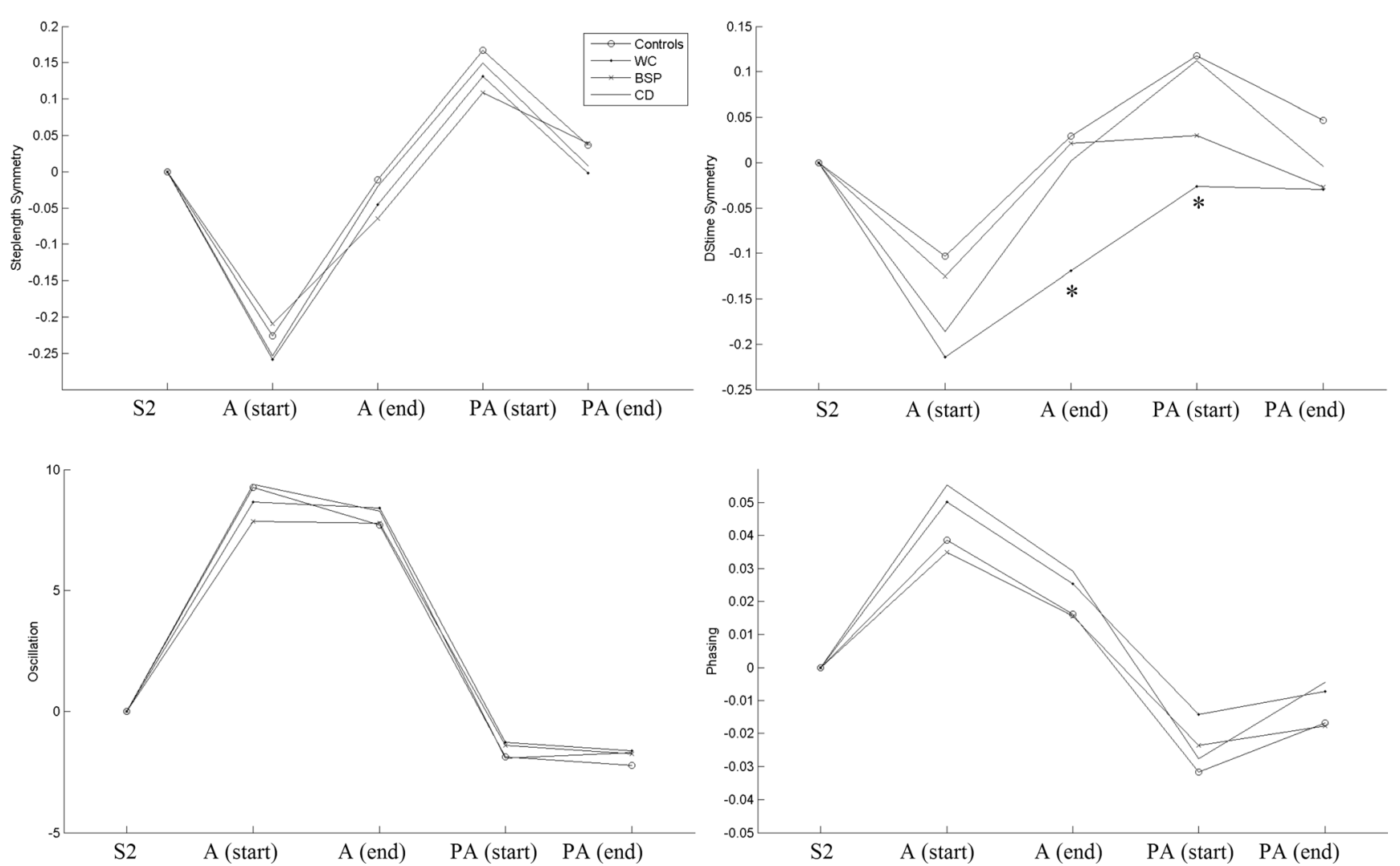

Fig. 3 Walking parameters (step length symmetry, double support time symmetry, oscillation, phasing) over the five periods with baseline (S2) subtraction, visualized per group (controls, WC, BSP, CD)

pathophysiological mechanisms in these different subtypes. In addition, we studied normal gait and tandem gait to investigate possible subtle gait differences between patients and controls.

The main limitation of our study is the relatively low number of patients per dystonia subgroup. As movement data was automatically generated from marker position data using custom written software, bias from non-blinded evaluations is expected to be small. Our results need to be confirmed by others and we encourage further studies of cerebellar motor learning in primary dystonia.

\section{Normal and Tandem Gait}

This study of gait firstly shows that patients with blepharospasm (BSP) and cervical dystonia (CD) prefer a lower walking speed and have significantly lower balance confidence compared to healthy controls (C) and patients with writer's 


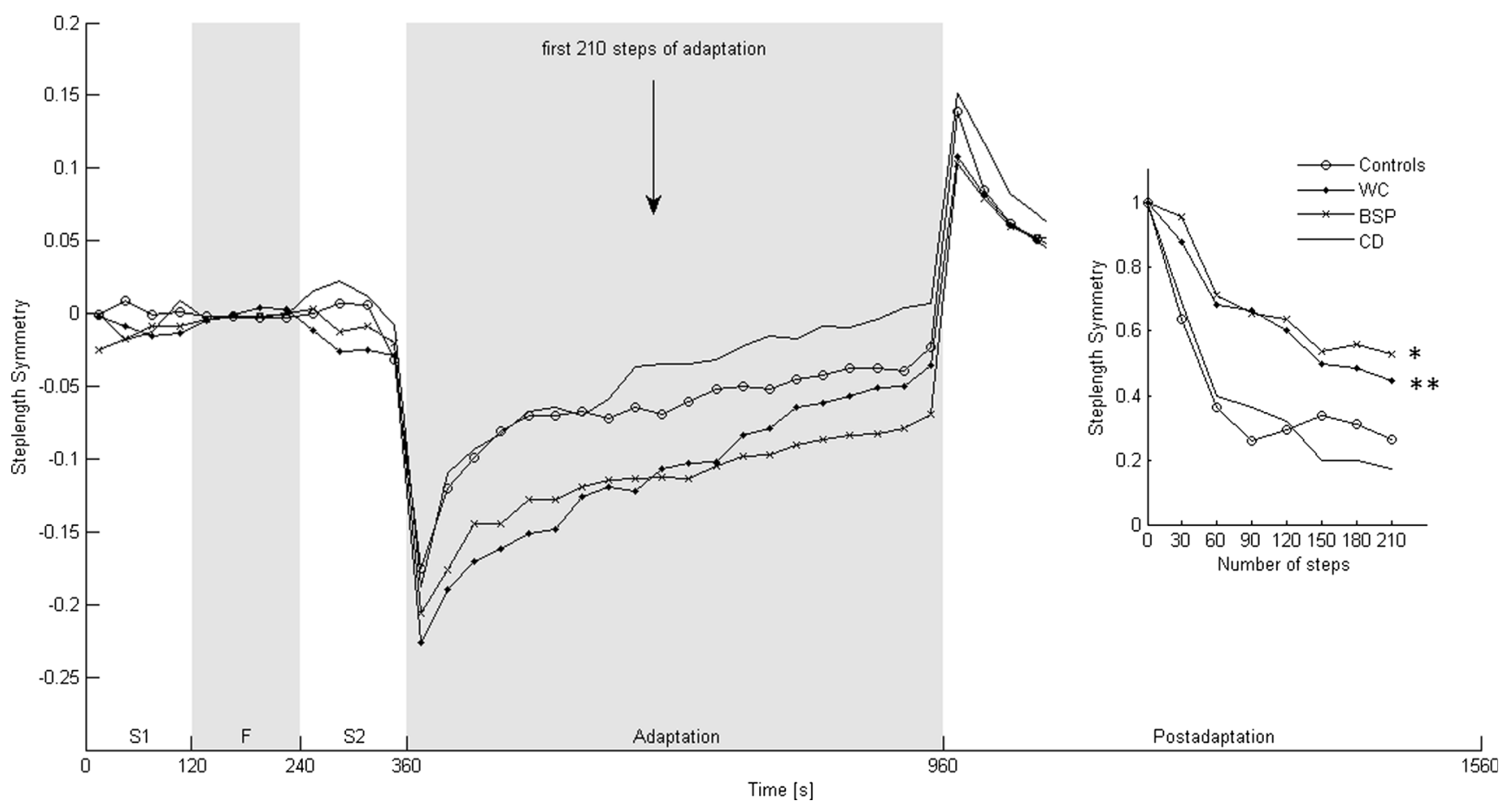

Fig. 4 Larger picture: Raw data of mean step length symmetry per $30 \mathrm{~s}$ for all four groups (controls, WC, BSP, CD) during the whole walking paradigm: slow walking $S 1$ (2 min), fast walking $F$ (2 min), slow walking $S 2$ (2 $\mathrm{min})$, adaptation (10 $\mathrm{min})$ and post-adaptation (10 $\mathrm{min})$. The arrow indicates the first 210 steps of adaptation as used in analysis of the adaptation rate. Smaller inset: Step length symmetry averaged in 7 bins of 30 steps; rescaled by their respective starting point cramp (WC). During normal walking, BSP patients differed significantly from $\mathrm{WC}$ on the ataxia ratio, reflecting less constant execution of movement in BSP that might result from cautious walking. Therefore, subtle kinematic gait abnormalities might exist in some forms of primary focal dystonia, but none of the gait abnormalities we observed were considered sufficiently indicative of cerebellar dysfunction, as in particular there were no tandem gait difficulties. Other explanations in the BSP group could be reduced visual input, or that - as mean age of BSP patients was slightly higher than the other three groups - there is more of an age-related reduction in walking speed [13]. For $\mathrm{CD}$, the explanation for a reduced balance confidence might lie in the abnormal head posture during walking, or in abnormal vestibular input as a consequence of this [14].

\section{Sensorimotor Adaptation}

Multiple abnormalities where observed in BSP and WC patients during split-belt walking.

Feed forward sensorimotor adaptation is used to adjust movements over minutes to hours in response to a certain perturbation, which leads to storage of a new motor pattern and therefore to aftereffects when the perturbation is removed. When testing the beginning of post-adaptation against zero to see if significant aftereffects (and therefore adaptation) had occurred, P-beginning did not significantly differ from zero for step length, double support time, or oscillation in BSP patients. In WC patients, P-beginning did not significantly differ from zero for double support time and phasing.

When testing split-belt walking during the five different periods of the experiment, significant differences were observed for DS time values with WC patients showing abnormal adaptation.

The rate of adaptation of step length symmetry was significantly different between patient groups, as WC and BSP patients displayed a slower rate of adaptation compared to patients with CD and healthy controls. The speed of step length symmetry adaptation can be influenced by changing both spatial (oscillation) and temporal (DS time, phasing) parameters of walking. So, for WC patients, a significant slower rate of adaptation probably resulted from abnormalities in temporal adaptation (DS time and phasing), whereas slower adaptation in BSP patients seems to be attributable to abnormalities in both spatial (oscillation) and temporal adaptation (double support time). The presently observed deficits in adaptations may be further indicators of cerebellar dysfunction in dystonia. Patients with cerebellar degeneration show impairments in both the spatial and temporal domains of splitbelt adaptation [3]. However, differential modulation of spatial and temporal parameters by conscious correction or by cerebellar transcranial direct current stimulation $[8,15]$ 
suggests that there are different neural substrates for the spatial versus temporal components. It has been hypothesized that the pontocerebellum (which projects to cortical regions) could play a stronger role in the spatial control of locomotion and that the spinocerebellum (projecting to the vestibulospinal and reticulospinal tracts) might be more involved in temporal coordination. Interestingly, patients with $\mathrm{WC}$ and BSP showed different abnormalities in these adaptation parameters, whereas patients with $\mathrm{CD}$ were not significantly impaired on this task. This might suggests different patterns, in terms of degree and area, of cerebellar malfunctioning in these different forms of primary dystonia.

We have recently demonstrated abnormal EBCC in CD patients [2] but found no clear abnormalities in this split-belt motor learning paradigm. This could be due to the fact that cerebellar dysfunction in $\mathrm{CD}$ is not as widespread as in other forms of focal dystonia. Of interest, Schwingenshuh et al. also observed similar levels of motor adaptation in $\mathrm{CD}$ patients and controls using a different task, although they did observe that patients might use a different strategy to complete a motor sequence learning task. They did not test other forms of dystonia [16].

In summary, we have demonstrated abnormal sensorimotor adaptation with the split-belt paradigm in patients with blepharospasm and writer's cramp. This reinforces the current concept of cerebellar dysfunction in primary dystonia and indicates that this abnormality extends beyond more pure forms of cerebellum-dependent associative motor learning paradigms. However, the finding of normal adaptation in cervical dystonia patients indicates that the pattern of cerebellar dysfunction is slightly different for the various forms of primary focal dystonia, but also argues against actual cerebellar pathology as a primary driving force in dystonia.

\begin{abstract}
Acknowledgements BSH and LCV are supported by a grant from the Prinses Beatrix Spierfonds. BvdW received research support from the Prinses Beatrix Spierfonds, Netherlands Brain Foundation, Gossweiler Foundation, the Royal Dutch Society for Physical Therapy, the Radboud University Medical Centre, and the Biobanking and Biomolecular Resources Research Infrastructure (BBMRI-NL). VW is supported by a Netherlands Organization for Scientific Research Veni Research Grant (916.10.106). The work described here was supported by a grant of the Prinses Beatrix Spierfonds to BvdW.
\end{abstract}

Conflict of Interest The authors report no financial (or other) conflict of interests.

\section{References}

1. Teo JT, van de Warrenburg BP, Schneider SA, Rothwell JC, Bhatia KP. Neurophysiological evidence for cerebellar dysfunction in primary focal dystonia. J Neurol Neurosurg Psychiatry. 2009;80(1):80-3. Epub 2008/12/19.

2. Hoffland BS, Kassavetis P, Bologna M, Teo JT, Bhatia KP, Rothwell $\mathrm{JC}$, et al. Cerebellum-dependent associative learning deficits in primary dystonia are normalized by rTMS and practice. Eur J Neurosci. 2013;38(1):2166-71. Epub 2013/04/05.

3. Morton SM, Bastian AJ. Cerebellar contributions to locomotor adaptations during splitbelt treadmill walking. J Neurosci Off J Soc Neurosci. 2006;26(36):9107-16. Epub 2006/09/08.

4. Forssberg H, Grillner S, Halbertsma J, Rossignol S. The locomotion of the low spinal cat. II. Interlimb coordination. Acta Physiol Scand. 1980;108(3):283-95. Epub 1980/03/01.

5. Vasudevan EV, Torres-Oviedo G, Morton SM, Yang JF, Bastian AJ. Younger is not always better: development of locomotor adaptation from childhood to adulthood. J Neurosci Off J Soc Neurosci. 2011;31(8):3055-65. Epub 2011/03/19.

6. Fasano A, Herzog J, Raethjen J, Rose FE, Muthuraman M, Volkmann J, et al. Gait ataxia in essential tremor is differentially modulated by thalamic stimulation. Brain. 2010;133(Pt 12):3635-48. Epub 2010/10/12.

7. Stolze H, Petersen G, Raethjen J, Wenzelburger R, Deuschl G. The gait disorder of advanced essential tremor. Brain. 2001;124(Pt 11): 2278-86. Epub 2001/10/24.

8. Malone LA, Bastian AJ. Thinking about walking: effects of conscious correction versus distraction on locomotor adaptation. J Neurophysiol. 2010;103(4):1954-62. Epub 2010/02/12.

9. van Heuvelen MJ, Hochstenbach J, de Greef MH, Brouwer WH, Mulder T, Scherder E. Is the activities-specific balance confidence scale suitable for Dutch older persons living in the community? Tijdschr Gerontol Geriatr. 2005;36(4):146-54. Epub 2005/10/01.

10. Reisman DS, Block HJ, Bastian AJ. Interlimb coordination during locomotion: what can be adapted and stored? J Neurophysiol. 2005;94(4):2403-15. Epub 2005/06/17.

11. Musselman KE, Patrick SK, Vasudevan EV, Bastian AJ, Yang JF. Unique characteristics of motor adaptation during walking in young children. J Neurophysiol. 2011;105(5):2195-203. Epub 2011/03/04.

12. Carbon M, Ghilardi MF, Argyelan M, Dhawan V, Bressman SB, Eidelberg D. Increased cerebellar activation during sequence learning in DYT1 carriers: an equiperformance study. Brain. 2008;131(1): 146-54. Epub 2007/10/20.

13. Samson MM, Crowe A, de Vreede PL, Dessens JA, Duursma SA, Verhaar HJ. Differences in gait parameters at a preferred walking speed in healthy subjects due to age, height and body weight. Aging. 2001;13(1):16-21. Epub 2001/04/09.

14. Munchau A, Bronstein AM. Role of the vestibular system in the pathophysiology of spasmodic torticollis. J Neurol Neurosurg Psychiatry. 2001;71(3):285-8. Epub 2001/08/21.

15. Jayaram G, Tang B, Pallegadda R, Vasudevan EV, Celnik P, Bastian A. Modulating locomotor adaptation with cerebellar stimulation. J Neurophysiol. 2012;107(11):2950-7. Epub 2012/03/02.

16. Katschnig-Winter P, Schwingenschuh P, Davare M, Sadnicka A, Schmidt R, Rothwell JC, et al. Motor sequence learning and motor adaptation in primary cervical dystonia. Journal of clinical neuroscience : official journal of the Neurosurgical Society of Australasia. 2013. Epub 2014/01/15. 